\title{
Metabolic targeted therapy of cancer: current tracer technologies and future drug design strategies in the old metabolic network
}

\author{
László G. Boros \\ SIDMAP, LLC and Los Angeles Biomedical Research Institute at Harbor-UCLA Medical Center, 10021 Cheviot Drive, \\ Los Angeles, CA 90064, USA
}

Received 23 August 2004; accepted 31 August 2004

\begin{abstract}
Targeted drugs tailored against genes and signaling proteins have formed the new era termed Targeted Therapies. Although the field is relatively young, since only about 5 years ago clinical trials started showing promise, there have are already been significant setbacks due to drug resistance caused by point mutations, alterations in gene expression or complete loss of target proteins with disease progression. Although new drugs are continuously designed and tried, it seems inevitable that genetic and signal protein targets pose too broad flexibility and variability, often changing target characteristics and thus escape treatments turning "magic bullets" into rather "wondering bullets". This is especially true in cancer, where old and new targeted therapies continue to fail and the most recent ones do not offer much improvement on clinical outcome parameters. Metabolic targeted therapies are aimed at control points of the metabolic network by targeting particular enzymes of major macromolecule synthesis pathways in cancer. This review summarizes the potential benefits of targeted therapies in the metabolic network as applied with genetic and proteomic approaches. The metabolic target approach is most efficient if and when pathway flux information is available for drug target development using the stable isotope based dynamic metabolic profile (SIDMAP) of tumor cells, in vitro or in vivo.
\end{abstract}

KEY WORDS: intermediary metabolism; stable isotope tracers; drug design; cancer.

\section{Introduction}

Tumor cells inherently possess various mechanisms to initiate and sustain any one of the following phenotypes: 1 . proliferative; 2 . differentiated; 3 . transformed; 4. cycle arrested; 5. necrotic; and 6. apoptotic (Boros et al., 2002a). Advanced and therapy resistant tumors share one common phenotype characterized by rapid proliferation, poor differentiation - increased transformation, as well as multiple drug and apoptosis resistance. They also exhibit a high rate of metabolism using glucose as the primary substrate (Pitot and Jost, 1967; Schwartz et al., 1986; Boekhorst et al., 1995; Smith, 1998). Therefore, it is likely that tumor cells' sensitivity to exogenous and endogenous factors determining growth and differentiation is deeply hidden in the metabolic network supplying essential substrates for de novo macromolecule synthesis and energy production. The rate of proliferation is closely associated with the rate of de novo macromolecule synthesis, such as RNA, DNA, amino acids and fatty acids (Eigenbrodt et al., 1992). These complex molecules, which eventually become structural components of new and old progenies of tumor cells, are synthesized from small molecular weight substrates, such as glucose, short chain fatty acids and amino acids in an inter-

\footnotetext{
*To whom correspondence should be addressed.

E-mail: lboros@sidmap.com
}

connected and complex metabolic network. All pathways in the network depend on one another via substrate sharing and channeling, and by regenerating shared co-factors that participate in oxidative degradation and reductive synthesis simultaneously. Such relationship is evident between direct glucose oxidation in the pentose cycle and de novo fatty acid synthesis, where part of the reduced $\mathrm{NADP}^{+}$pool is regenerated allowing the irreversible glucose-6-phosphate dehydrogenase reaction to proceed for the synthesis of five carbon sugars (Kuhajda, 2000; Baron et al., 2004). In turn, the reducing $\mathrm{NADP}^{+}$equivalent is used during reductive de novo synthesis of fatty acids, their chain elongation and de-saturation, allowing distant metabolic network processes to proceed in a well-controlled and synchronized fashion. Cell phenotypes and their sensitivity to apoptosis, which is the focus of this review; demonstrate differences in their respective stable isotope-labeled dynamic metabolic profiles (SIDMAP) of cross-regulated metabolic pathways in the network. The models discussed herein include therapy resistant inflammatory breast cancer cells in comparison with apoptosis sensitive human fibroblasts and therapy sensitive pancreatic tumor cells. I herein argue that targeted therapies of the metabolic network may overcome difficulties of drug design related to the enormous variability of the ever changing pool of genetic and proteomic targets in cancer ( $\mathrm{Hu}$ and Kavanagh, 2003; Cowan-Jacob et al., 2004; Shah et al., 2004; Guillemard 
and Saragovi, 2004). However, one has to first learn how to trace and read the map of the uniquely altered metabolic network of tumor cells in order to design new targeted therapies within.

\section{Targeted therapies of cancer using genetic and proteomic targets}

Targeted drugs are designed for a single or a very narrow range of genetic or protein targets. Although they are extremely effective to treat narrow cancer cell populations with a very favorable low toxicity profile towards the host, their main limitations are high dependence on a single highly modifiable target and the rapid development of resistance based on four major mechanisms: 1. decrease in target protein expression; 2. mutations in target proteins; 3. loss of target gene and/or construct due to clonal selection; and 4. increased drug transport from targeted cells. These limitations have produced significant delays, cost accumulations or disappointments with almost all targeted drugs designed so far.

\section{Efforts in targeted drug design against genetic and protein targets in cancer}

Targeted therapies have continuously been developed and explored for the treatment of human cancer. Monoclonal antibodies, for example epidermal growth factor monoclonal antibodies (EGF $\mathrm{mABs}$ ), and small molecule receptor antagonists have offered high specificity against cytokines and cell surface receptors to inhibit growth signaling. The main challenges are host immune response and increased cytokine production, leading to low response rate especially in advanced refractory cancers (Dancey and Freidlin, 2003). Another approach is to conjugate toxins or radioisotopes for the treatment of leukemia, which offers targeted delivery of high efficacy drugs. The delivery of these toxic agents is ligand dependent and therefore in clinical trials low response rate and recurrence still pose challenges (Sievers, 2000; Nemecek and Matthews, 2003). Antisense oligonucleotides are gene expression modifying short nucleic acid sequences, where the delivery system is still not resolved because of severe host response to viral vectors. The most severe side effects are hemolytic anemia, renal failure and delayed immune reactions such as anasarca, which held up further clinical tests (Rudin et al., 2001). Immunoliposome-encapsulated drugs are aimed for targeted delivery of protected antibodies. Their main disadvantages are moderate stability and the inability of the carrier to extravasate, or to leave the circulatory system, leading to low efficacy and high failure rate in clinical trials (Matzku et al., 1990). Small molecule inhibitors, such as imatinib (STI-571), target single oncogenic protein constructs, however recurrence with blast disease and increasing drug resistance in chronic myeloid leukemia is an emerging and severe problem (Hofmann et al., 2004).

It is evident that although the targeting of narrow oncogenic constructs with "magic bullets" is an old approach that raised high expectations, multiple mechanisms slipping the target continuously off site in the ever changing genetic and proteomic maze of targets make this approach ever more expensive and less effective. One solution is to redesign drugs with altered structure to hit a new target range (Shah et al., 2004), but this approach is controversial, again, given the difficulties and costs involved in repeated clinical trials for each new drug, targeting slightly mutated proteins or increasing expression of genes. This will likely exhaust research and development budgets of even large drug companies on the long run, as the cost of targeted therapy drug design is enormous, as compared to conventional treatments, while the return is strenuous due to narrow groups and limited number of patients who benefit from them. It is also evident that health insurance companies will not be able to cover the ever growing costs of targeted therapies, lasting for as long as they would be necessary to keep the ever changing genetic and protein profiles of tumor growth under control (Danzon and Towse, 2002). Considering even a few possible meaningful oncogenic permutations as expected from gene and protein variations, targeted therapies against the genetic and proteomic network may well be the most expensive medicine has yet taken with little in return regarding cancer disease outcome or improving population health.

\section{Metabolomics: the study of the transformed metabolic network in cancer}

Metabolomics is a new field in medicine addressing and developing platforms which presumably will achieve better understanding and allowing more effective drug design against many human diseases, including cancer (Schmidt, 2004). Metabolomics as a tool is designed around quantitative metabolite level measurement and ratios, which are mined typically, using several pattern recognition techniques, including principal components analysis and hierarchical cluster analysis. This combined approach is being termed metabolomics and foresights into the field predict a strong competing role in medicine to genetics and proteomics. While there is still important reliance on functional genomics to further elucidate the role of genes and their protein products in human cancer, there is also an increasing necessity to define and understand how the genetic and protein networks conspire with the metabolism of particular tumor phenotypes (Griffin, 2004). Although it is known that metabolic networks also vary in their substrate utilization patterns and flux distribution reflecting cell 
function and phenotype, their control points have strictly been preserved throughout evolution and are very reliable drug targets considering the limited number of enzyme isoforms as well as the limited number of major alternative metabolic routes, which are mostly known. Metabolism is an old field with new potentials to assist drug development, although one has to first learn how to navigate among the many interconnected pathways, how to trace and define targets, and how to determine and utilize existing control among distant metabolic pathways in the same network (Boros et al., 2003c).

\section{Stable isotope labeled metabolic network and sensitivity to apoptosis}

\subsection{Apoptosis sensitive cells heavily depend on non-oxidative pentose cycle metabolism while lacking de novo fatty acid synthesis}

The double tracer approach using stable isotope labeled glucose is particularly effective in revealing detailed substrate flow and distribution patterns in the complex metabolic network of human cells. Applications in cancer have greatly assisted us to understand growth controlling mechanisms in the transformed network (Boros et al., 2002b, Boros 2003a). SIDMAP has revealed that pentose cycle metabolism with lacking de novo fatty acid synthesis makes human fibroblasts extremely vulnerable to apoptosis when thiamine, cofactor of the transketolase non-oxidative pentose cycle enzyme, becomes limited due to defective high affinity thiamine transport (Green 2003; Boros et al., 2003b). Thiamine transport deficient human fibroblasts readily undergo apoptosis in culture with no rescue mechanism in place as they lack de novo fatty acid synthesis and therefore posses limited reserves of the oxidized form of $\mathrm{NADP}^{+}$, which is the sole hydrogen acceptor during oxidative pentose synthesis from glucose in the cycle. Similarly, pancreatic adenocarcinoma cells (MIA PaCa) show limited growth and in response to pentose cycle inhibitors (Boros et al., 1997) and possess a relatively low rate $(20 \%)$ de novo fatty acid synthesis and turnover during the $72 \mathrm{~h}$ treatment period (Boros et al., 2001a, 2001b). Figure 1 demonstrates metabolic pathway substrate flow in apoptosis sensitive human cells using doubly labeled glucose as the tracer.

\subsection{Apoptosis resistant cells heavily depend on oxidative pentose cycle metabolism by maintaining high rate of de novo fatty acid synthesis and turnover}

The SIDMAP of therapy and apoptosis resistant tumor cells shown on figure 1 is different from that of therapy sensitive cells shown on Figure 2, which illustrates the metabolic profile of therapy resistant

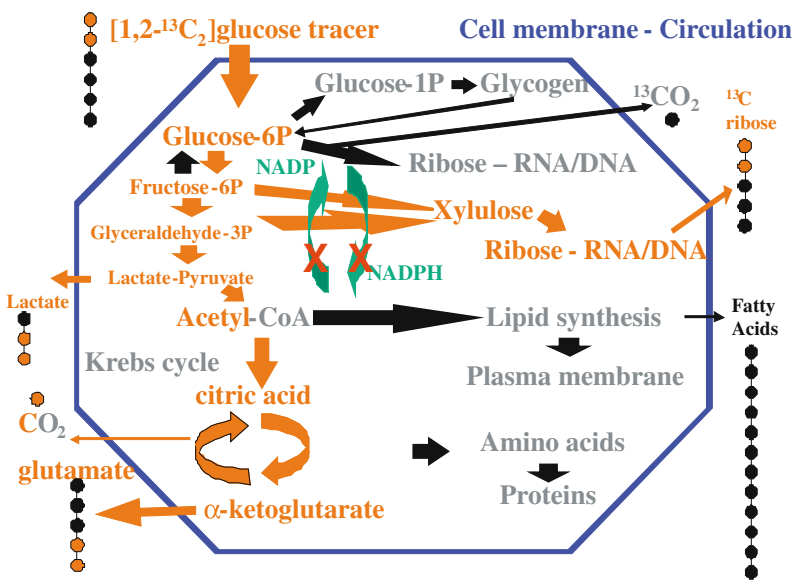

Figure 1. SIDMAP of apoptosis sensitive human cells. Red arrows indicate routes of ${ }^{13} \mathrm{C}$ tracer glucose substrate carbons (red filled circles) in the metabolic network. The heavy use of glucose carbons via the pentose cycle in human fibroblasts (TRMA cells) is primarily via the non-oxidative route, while the oxidative pathway is limited due to low NADP-NADPH cycling and fatty acid synthesis. Black arrows indicate less active pathways. Human fibroblasts with high affinity thiamine transport deficiency readily undergo spontaneous apoptosis (Boros et al., 2000b) and MIA PaCa show sensitivity and slow growth to non-oxidative pentose cycle inhibitors (Boros et al., 1997).

inflammatory breast cancer cells indicating intense tracer accumulation into fatty acids.

The main difference is the rate at which they synthesize medium and long chain saturated fatty acids up to the 16 carbon chain length palmitate and consequently elongate it to the 18 carbon length stearate and further into C:20-C:26 species. They also posses high fatty acid chain desaturase activity, further oxidizing NADPH and allowing the oxidative branch of the pentose cycle to operate under drug treatment. This is especially important when nucleic acid synthesis inhibitors are targeting either branch of the pentose cycle, the operation of these alternative synthesis routes is essential for the survival of tumor cells and to endure apoptosis inducing drugs and signals.

\section{Future metabolic drug design scenarios based on what we know already}

Genetics and proteomics generated enough data in the last two decades that targeted therapies against unique genes and proteins are entering clinical practice for the treatment of cancer. Significant resistance to new targeted therapies as a clinical problem appeared in a rather quick and frequent manner, bringing new challenges back to the laboratory. As metabolic tracer data accumulates and the operation of the transformed metabolic network of tumor cells is slowly revealed, new opportunities have arisen via metabolic targeted therapies. The current challenges are several, first of all more data are needed and need to be correlated with tumor phenotypes, biological behavior and metabolic network 


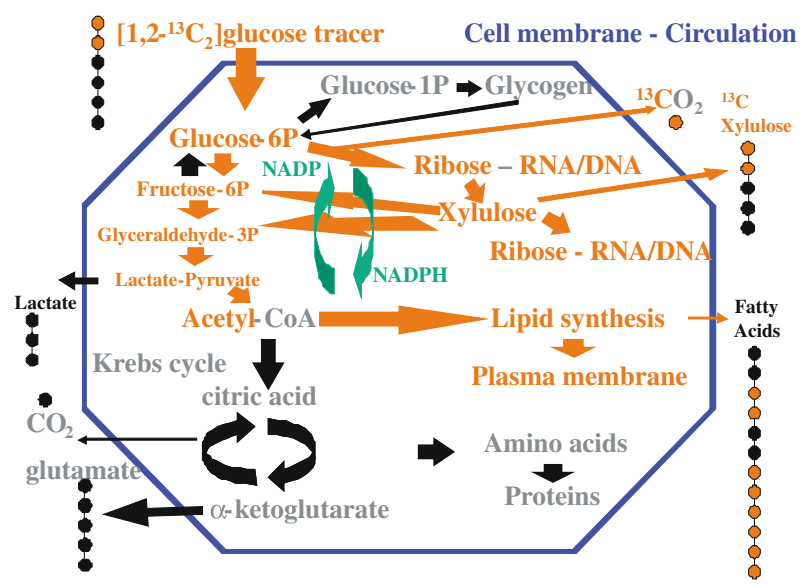

Figure 2. SIDMAP of apoptosis resistant tumor cells. Red arrows indicate routes of ${ }^{13} \mathrm{C}$ tracer glucose substrate carbons (red filled circles) in the metabolic network. NADPH-NADP cycling is active and is compensating in response to non-oxidative pentose cycle inhibitor treatment. Black arrows indicate less active pathways. Inflammatory breast cancer cells exhibiting this SIDMAP are extremely durable, treatment resistant and aggressive. Although growth retardation is achieved, inflammatory breast cancer cells can not be forced into apoptosis even when the toxic glucose derivative 2-deoxy-D-glucose derivative is given in high doses $(5 \mathrm{mM})$ (Boren et al., 2001).

characteristics. Secondly, it is likely that metabolic targeted therapies against tumors will have to be aimed at multiple sites and control enzymes in the network due to the fact that metabolic networks are interconnected and alternative synthesis pathways are common (that is to say a metabolic network exists). Thirdly, individual SIDMAPs of tumor cells and host organs will likely help tailoring metabolic targeted drugs to individual tumor growth characteristics in the host. Based on what is known so far, it can be predicted that limited de novo fatty acid synthesis of a given tumor will allow pentose cycle inhibitors to work effectively, while tumors possessing high rate of fatty acid turnover have to be targeted with a combined approach using fatty acid synthase, chain elongase and desaturase inhibitors, along with conventional drugs targeting pentose cycle synthesis, nucleic acid backbone sugar production, RNA synthesis, DNA replication and consequently cell proliferation. Tumor SIDMAPs can easily be determined both in vitro and in vivo, using non-invasive, nonradiating and natural sugar tracers for both diagnostic and metabolic targeting purposes.

\section{Conclusions}

As metabolic profiling allows new targets to be discovered, their promise is that these targets will have significantly far less flexibility and variability to escape treatment than do genetic and signal protein targets. This is based on the fact that structures of metabolic enzymes and hierarchies of metabolic networks are wellpreserved throughout evolution and among species, and tumor cells have to adhere to these hierarchies to survive. Regardless of the level of transformation and malignancy, tumor cells have to integrate and co-ordinate their metabolism with a complex host operating on a limited number of substrates and co-operative futile cycles. It is evident that mutations in growth signal proteins make them hidden from drugs that target them without losing function. Many growth signals exist contemporarily, they initiate downstream effects that can become constitutively active (Boros, 2004) and they can maintain signaling by variations in gene expression. On the other hand, mutations in metabolic enzyme proteins, although may also make them to escape newly designed metabolic reaction targeting drugs, these mutations will also make them non-functional and defective to catalyze the metabolic reaction which the inhibitor tends to control. Over-expression of metabolic enzymes is rather a real threat to develop resistance against metabolic targeted therapies and this is where a combined approach of genomics, proteomics and metabolomics will change the future. The purpose of metabolomics in the new targeted era of drug design is to pinpoint targets in the fundamental components of cell function. These targets have very limited flexibility and variability to develop resistance by point mutations or structural/conformational changes, or any other mechanism that make genetic and protein targets weak and short-lived.

\section{Acknowledgment}

The Inflammatory Breast Cancer Research Foundation has provided funding for the research efforts outlined in the review.

\section{References}

Baron, A., Migita, T., Tang, D. and Loda, M. (2004). Fatty acid synthase: a metabolic oncogene in prostate cancer? J. Cell. Biochem. 91, 47-53.

Boekhorst, P.A., Lowenberg, B., van Kapel, J., Nooter, K. and Sonneveld, P. (1995). Multidrug resistant cells with high proliferative capacity determine response to therapy in acute myeloid leukemia. Leukemia 9, 1025-1031.

Boren, J., Cascante, M., Marin, S., et al. (2001). Gleevec (STI571) influences metabolic enzyme activities and glucose carbon flow toward nucleic acid and fatty acid synthesis in myeloid tumor cells. J. Biol. Chem. 276, 37747-37753.

Boros, L.G., Puigjaner, J., Cascante, M., et al. (1997). Oxythiamine and dehydroepiandrosterone inhibit the nonoxidative synthesis of ribose and tumor cell proliferation. Cancer Res. 57, 42424248 .

Boros, L.G., Bassilian, S., Lim, S. and Lee, W.N. (2001a). Genistein inhibits non-oxidative ribose synthesis in MIA pancreatic adenocarcinoma cells: a new mechanism of controlling tumor growth. Pancreas 22, 1-7.

Boros, L.G., Lapis, K., Szende, B., et al. (2001b). Wheat germ extract decreases glucose uptake and RNA ribose formation but in- 
creases fatty acid synthesis in MIA pancreatic adenocarcinoma cells. Pancreas 23, 141-147.

Boros, L.G., Lee, W.N. and Go, V.L. (2002a). A metabolic hypothesis of cell growth and death in pancreatic cancer. Pancreas 24, 2633.

Boros, L.G., Cascante, M. and Lee, W.N. (2002b). Metabolic profiling of cell growth and death in cancer: applications in drug discovery. Drug Discov. Today 7, 364-372.

Boros, L.G., Brackett, D.J. and Harrigan, G.G. (2003a). Metabolic biomarker and kinase drug target discovery in cancer using stable isotope-based dynamic metabolic profiling (SIDMAP). Curr. Cancer Drug Targets 3, 445-453.

Boros, L.G., Steinkamp, M.P., Fleming, J.C., Lee, W.N., Cascante, M. and Neufeld, E.J. (2003b). Defective RNA ribose synthesis in fibroblasts from patients with thiamine-responsive megaloblastic anemia (TRMA). Blood 102, 3556-3561.

Boros, L.G., Cascante, M. and Lee, W.-N.P. (2003c). Stable isotopebased dynamic metabolic profiling in disease and health in Harrigan, G.G. and Goodacre, R. (Eds), Metabolic profiling: its role in biomarker discovery and gene function analysis. Kluwer Academic Publishers, Boston.

Boros L.G. (2004). Metabolic profile of inflammatory breast cancer: aiding diagnosis and treatment. George Washington University and the IBC Research Foundation co-sponsored "IBC Mini Symposium" hosted by George Washington University: http:// www.ibcresearch.org/ibcminisymposium/.

Cowan-Jacob, S.W., Guez, V., Fendrich, G., et al. (2004). Imatinib (STI571) resistance in chronic myelogenous leukemia: molecular basis of the underlying mechanisms and potential strategies for treatment. Mini Rev. Med. Chem. 4, 285-299.

Dancey, J.E. and Freidlin, B. (2003). Targeting epidermal growth factor receptor - Are we missing the mark?. Lancet 362, 6264.

Danzon, P. and Towse, A. (2002). The economics of gene therapy and of pharmacogenetics. Value Health 5, 5-13.

Eigenbrodt, E., Reinacher, M., Scheefers-Borchel, U., Scheefers, H. and Friis, R. (1992). Double role for pyruvate kinase type M2 in the expansion of phosphometabolite pools found in tumor cells. Crit. Rev. Oncog. 3, 91-115.

Green, R. (2003). Mystery of thiamine-responsive megaloblastic anemia unlocked. Blood 102, 3464-3465.
Griffin, J.L. (2004). Metabolic profiles to define the genome: can we hear the phenotypes? Philos. Trans. R. Soc. Lond. B Biol. Sci. 359, 857-871.

Guillemard, V. and Saragovi, H.U. (2004). Novel approaches for targeted cancer therapy. Curr. Cancer Drug Targets 4, 313-326.

Hofmann, W.K., Komor, M., Hoelzer, D. and Ottmann, O.G. (2004). Mechanisms of resistance to STI571 (Imatinib) in Philadelphiachromosome positive acute lymphoblastic leukemia. Leuk. Lymphoma 45, 655-660.

Hu, W. and Kavanagh, J.J. (2003). Anticancer therapy targeting the apoptotic pathway. Lancet Oncol. 4, 721-729.

Kuhajda, F.P. (2000). Fatty-acid synthase and human cancer: new perspectives on its role in tumor biology. Nutrition 16, 202-208.

Matzku, S., Krempel, H., Weckenmann, H.P., Schirrmacher, V. and Sinn, H. Stricker, H.1990Tumor targeting with antibodycoupled liposomes: failure to achieve accumulation in xenografts and spontaneous liver metastases. Cancer Immunol. Immunother. 31, 285-291.

Nemecek, E.R. and Matthews, D.C. (2003). Use of radiolabeled antibodies in the treatment of childhood acute leukemia. Pediatr. Transplant. 3, 89-94.

Pitot, H.C. and Jost, J.P. (1967). Control of biochemical expression in morphologically related cells in vivo and in vitro. Natl. Cancer Inst. Monogr. 26, 145-166.

Rudin, C.M., Holmlund, J., Fleming, G.F., et al. (2001). Phase I Trial of ISIS 5132, an antisense oligonucleotide inhibitor of c-raf-1, administered by 24-hour weekly infusion to patients with advanced cancer. Clin. Cancer Res. 7, 1214-1220.

Schmidt, C. (2004). Metabolomics takes its place as latest up-andcoming "omic" science. J. Natl. Cancer Inst. 96, 732-734.

Schwartz, A.G., Pashko, L. and Whitcomb, J.M. (1986). Inhibition of tumor development by dehydroepiandrosterone and related steroids. Toxicol. Pathol. 14, 357-362.

Shah, N.P., Tran, C., Lee, F.Y., Chen, P., Norris, D. and Sawyers, C.L. (2004). Overriding imatinib resistance with a novel ABL kinase inhibitor. Science 305, 399-401.

Sievers, E.L. (2000). Targeted therapy of acute myeloid leukemia with monoclonal antibodies and immunoconjugates. Cancer Chemother. Pharmacol. 46, S18-S22.

Smith, T.A. (1998). FDG uptake, tumor characteristics and response to therapy: a review. Nucl. Med. Commun. 19, 97-105. 